\title{
Specific Antibody Detection in \\ Dogs with Filarial Infections
}

Deborah E. Joekel ', Simone Maier' ${ }^{1}$, Katja Huggel' ', Roland Schaper², Peter Deplazes ${ }^{1} \bowtie$

${ }^{1}$ Institute of Parasitology, University of Zurich, 8057 Zurich, Switzerland

2 Bayer Animal Health GmbH, 51368 Leverkusen, Germany

\section{Corresponding author:}

\section{Peter Deplazes}

Email: deplazesp@access.uzh.ch

\section{Abstract}

Dirofilariosis is a mosquito-transmitted disease of wild and domestic carnivores. Etiological diagnosis on canine Dirofilaria-infections is generally either based on the morphological or molecular characterization of microfilariae (L1), or in case of D. immitis-infection on the detection of circulating antigens shed by mature female worms. However, these tests do not detect infections during the long prepatent period of 182-238 days. We hereby present a monoclonal antibody based sandwich-ELISA used for on-plate purification of somatic antigen of adult D. immitis stages for the detection of antibodies against $D$. immitis and $D$. repens in dog sera. Sensitivity of the assay for $D$. immitis patent infections was calculated to be $93.8 \%$ (95\% CI: $79.2-99.2 \%$ ), and for patent D. repens-infections $100 \%$ (95\%
CI: $81.9-100 \%)$. Specificity was determined to be $98.6 \%$ (95\% CI: $92.2-100 \%$ ) with sera of 69 dogs from a non-endemic area. Cross-reactions against other nematodes such as Acanthocheilonema and Dipetalonema spp. (50\% and $66.7 \%$, respectively), Crenosoma vulpis (16.7\%), Capillaria aerophila $(14.3 \%)$ and naturally, but not experimentally infected dogs with Angiostrongylus vasorum $(14.3 \%)$ were present. However, in all positive dogs a residence in a filarial endemic area cannot be excluded. No positive reactions could be shown in experimentally infected dogs with Toxocara canis, Ancylostoma caninum and Trichuris vulpis. Dogs experimentally infected with $D$. repens showed seroconversion between 24-80 days post inoculation (dpi), far earlier than beginning of patency (189-259 dpi). Accordingly, the presented ELISA could be a supplementary or alternative tool for 
the diagnosis of Dirofilaria-infected dogs in low or non-endemic areas to document the contact rate and infection pressure.

\section{Introduction}

The causative agents of dirofilariosis are nematodes of the genus Dirofilaria, parasitizing predominantly wild carnivores of different species but also affecting companion animals such as dogs and rarely cats and ferrets. Dirofilariosis is a zoonotic, vector-borne disease and its transmission is dependent on the occurrence of competent vector species of the genera Culex spp., Aedes spp. and Anopheles spp. (Ludlam et al. 1970, Cancrini et al. 2007). Apart from Switzerland, Germany, Austria and Scandinavian countries, the often apathogenic agent $D$. repens is (low-)endemic in Europe by now. The clinically more relevant species $D$. immitis, commonly known as "heartworm", occurred sporadically in Central and Western Europe, whereas southernmost countries are assumed to be (highly) endemic (Baneth et al. 2016). Increasing European animal transfers of infected dogs from the Mediterranean as well as travelling habits of people including their dog companions to endemic areas are common practice and therefore problematic in the context of disease distribution (Genchi et al. 2011). Global trade is furthermore of increasing concern when vectors are accidentally trafficked, leading to new vector habitats or the possibility of parasite transmission and distribution via invasive mosquito species to populations of susceptible hosts in hitherto non-endemic regions (Silaghi et al. 2017).

There are several approaches to diagnose D. immitis-infection intra vitam in affected dogs, whereas no test is currently able to detect larval infections. Case history combined with radiography is used in veterinary practice for presumptive evidence of heartworm disease in dogs due to (pathognomonic) clinical and pathological signs. However, diagnosis based on these criteria only is assumed to be insensitive, since clinical signs vary from being absent, mild, moderate or severe (reviewed by Bowman and Atkins 2009). Beside molecular proof of DNA from microfilariae in a blood sample by PCR (Rishniw et al. 2006, Latrofa et al. 2012, Magnis et al. 2013), microfilariae can be detected from EDTA-blood after enrichment via Knott- (Knott 1939) or filter-method (Bell 1967) and can be differentiated by well described morphological criteria such as body length and shapes of front end and tail (Magnis et al. 2013). Furthermore, the distribution of acid phosphatase activity is used to distinguish between microfilariae of the genus Dirofilaria and from other filarial nematode genera such as Acanthocheilonema or Dipetalonema (Chalifoux et al. 1971, Peribáñez et al. 2001). It has to be pointed out that all diagnostic approaches mentioned above require a patent infection and since microfilarial periodicity was demonstrated, samples collected in the evenings (between 06.00-10.00 p.m.) usually contain higher values of microfilariae per milliliter blood (Di Cesare et al. 2013). However, occult (amicrofilaremic) infections lacking circulating microfilariae due to prepatency, unigender infection, anthelmintic treatment-induced adult sterility and infection in which microfilariae are killed by anthelmintics or by immune response are common (Rawlings et al. 1982, Bowman and Mannella 2011). The potential absence of microfilariae in heartworm-infected dogs led to serious diagnostic uncertainties and inaccuracies.

A substantial scientific achievement and the most common $D$. immitis diagnosis nowadays is based on the detection of antigens produced by mature females in the definitive host's blood (Weil 1987). Antigenemia during D. immitis infection generally occurs at the conclusion of the prepatent period approximately 5-7 months post inoculation with L3 larvae and persists for months-years during patency (Goodwin 1998). Various commercially available ELISAs detect infections with at least one adult female worm and are assumed to be highly sensitive (increasing with higher female heartworm burden and age of worms) and almost 
$100 \%$ specific (Atkins 2003, Lee et al. 2011). However, another study revealed cross-reactions with sera from dogs infected with Angiostrongylus vasorum in $3 / 6$ commercially available $D$. immitis test kits (Schnyder and Deplazes 2012). Furthermore, antibody-complexes due to e.g., chemotherapy (Drake et al. 2015) or other reasons leading to falsenegative results in current antigen-diagnosis were present in $7 \%$ of samples in a study from the United States of America (Velasquez et al. 2014). This phenomenon may be reverted by pre-treatment of sera with heat (Little et al. 2014, Ciucă et al. 2016). The aim of this study was the development of an ELISA to detect antibodies against Dirofilaria spp. infections as a supplementary or alternative tool to diagnose (larval, immature and patent) infections in dogs for e.g., epidemiological and surveillance studies in hitherto low- or non-endemic regions.

\section{Materials and Methods}

Mice experiments were conducted according to the Swiss guidelines for animal experimentation and approved by the Cantonal Veterinary Office of Zurich prior to the study (permission number 139/2015).

\section{Dog sera}

Sera from dogs proven positive for patent $D$. immitis $(\mathrm{n}=32)$ or $D$. repens $(\mathrm{n}=15)$ infection by evidence of microfilariae or necropsy results were included in this study. Furthermore, sera from three experimentally infected $D$. repens dogs (kindly provided by Bayer Animal Health GmbH, Leverkusen, Germany) that were tested positive for cutaneous adult worms at necropsy were used to determine the course of antibody development during infection (sampling days cf. Fig. 1) (Petry et al. 2015). Thirty-four sera from healthy blood donor dogs originated from a known Dirofilaria non-endemic area in Switzerland were used to determine the cut-off value (mean value plus 3 standard deviations). Specificity was calculated with randomly selected sera of 69 dogs presented at the Clinic for Small Animals and tested at the Clinical Laboratory of the Vetsuisse Faculty, University of Zurich, Switzerland, for various reasons excluding suspected parasitological infections (Schucan et al. 2012). Potential cross-reactions were tested with sera from dogs, experimentally infected with Toxocara canis $(\mathrm{n}=5)$, Ancylostoma caninum $(\mathrm{n}=4)$ and Trichuris vulpis $(\mathrm{n}=2)$ (last two mentioned were kindly provided by the Institute of Parasitology, University of Veterinary Medicine, Hannover); from experimentally $(n=10)$ and naturally $(n=7)$ infected dogs with Angiostrongylus vasorum (Schucan et al. 2012); from naturally infected dogs with Crenosoma vulpis $(\mathrm{n}=6)$ diagnosed by the presence of L1 in faeces (Barutzki and Schaper 2009); from naturally infected Italian dogs tested positive for the presence of Capillaria aerophila eggs $(\mathrm{n}=7)$ by PCR-coupled sequencing (Traversa et al. 2011); and from naturally infected dogs with Acanthocheilonema reconditum $(\mathrm{n}=10)$ and Dipetalonema dracunculoides $(\mathrm{n}=9)$ (Magnis et al. 2013).

\section{Antigens}

D. immitis adult E/S antigen was obtained from cultured worms (Deplazes et al. 1995), D. immitis/D. repens adult somatic antigen was prepared as described by Schucan et al. (2012). Protein concentrations were assessed by absorbance assay at $280 \mathrm{~nm}$ using a spectrometer (NanoDrop ${ }^{\circledR}$ ND-1000; Witec AG, Littau, Switzerland).

\section{Monoclonal antibodies}

Production of murine monoclonal antibodies (mAbs) against $D$. immitis adult male E/S antigen was based on a protocol already described by Schnyder et al. (2011). Antibody production of the fused cells was checked by ELISA (cf. Screening of mAbs (primary screening) in the next paragraph). Supernatants positive in the primary screening were subsequently checked for cross-reactivity by ELISA with A. vasorum somatic antigen $(10 \mu \mathrm{g} /$ $\mathrm{ml}$ ). Selected (A. vasorum-negative) clones were subcloned to assure that antibody production 


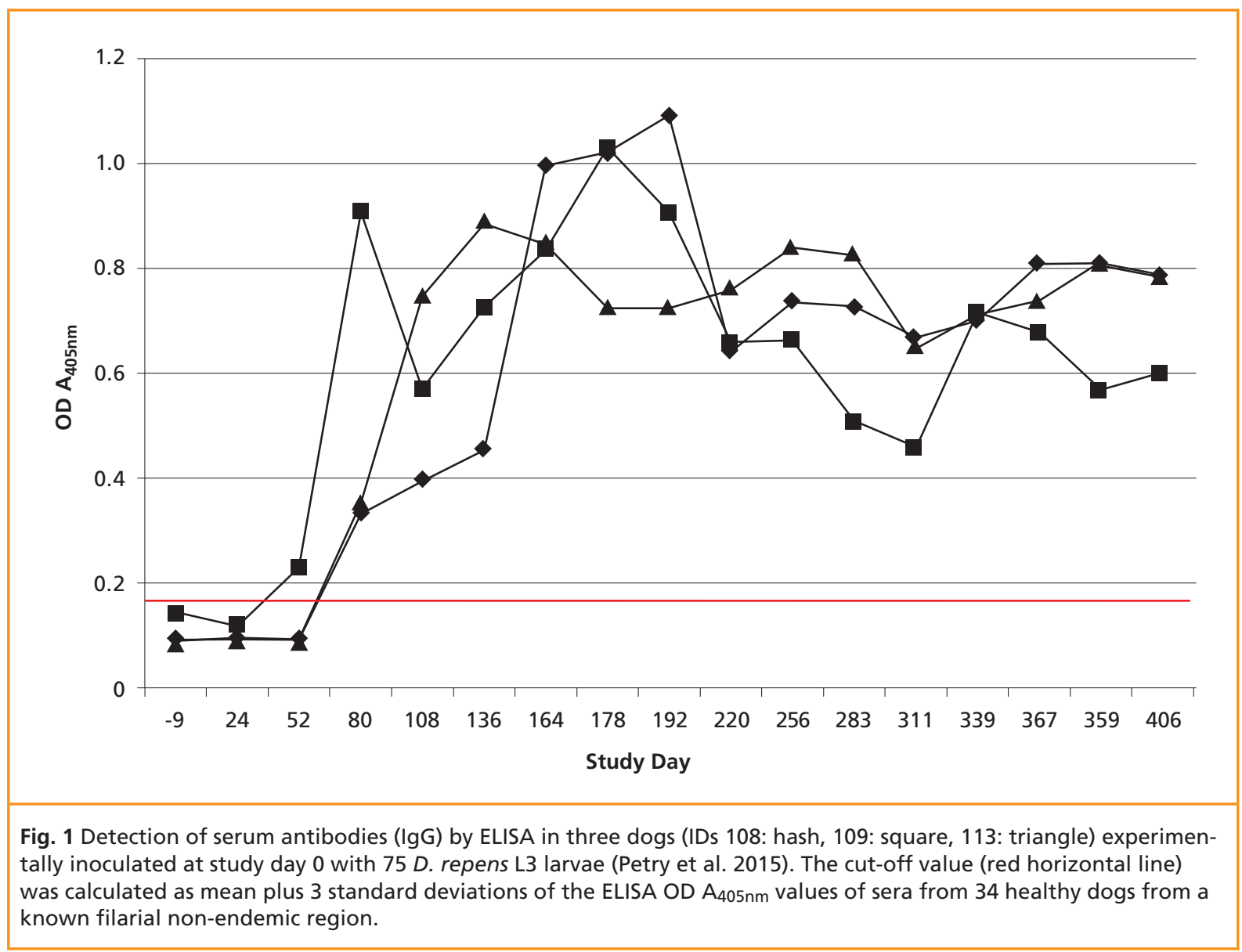

Table 1 Evaluation of sensitivity and specificity of the ELISA in defined canine sera. Cut-off determination: mean value of optical density $\left(A_{405 \mathrm{~nm}}\right)$ plus 3 standard deviations of 34 healthy dogs from a known Dirofilaria spp. non-endemic region.

\begin{tabular}{|c|c|c|}
\hline \multicolumn{2}{|c|}{ Sensitivity \% $(95 \% \mathrm{Cl})$} & Specificity $\%(95 \% \mathrm{Cl})$ \\
\hline D. immitis* & D. repens* & Random samples** \\
\hline$(\mathrm{n}=32)$ & $(\mathrm{n}=15)$ & $(\mathrm{n}=69)$ \\
\hline $93.8 \%(79.20-99.20)$ & $100 \%(81.90-100)$ & $98.6 \%(92.20-100)$ \\
\hline
\end{tabular}

$\mathrm{Cl}$ : Confidence interval

* Patent Dirofilaria-infections confirmed by the presence of microfilariae in EDTA-blood, or adult worms at necropsy. ** Sera of randomly selected dogs without suspicion of a parasitological disease tested at the Clinical Laboratory of the Vetsuisse Faculty of the University of Zurich.

was monoclonal. A clone mAbDi36/1 positive for $D$. immitis and D. repens adult somatic and $\mathrm{E} / \mathrm{S}$ antigen was cultivated and supernatants were concentrated using Amicon ${ }^{\circledR}$ Ultra-15 Centrifugal Filters Ultrace ${ }^{\circledR}{ }_{-} 30 \mathrm{~K}$ (Merck Millipore Ltd., Ireland) and the mAbs were subsequently purified by
Magne $^{\mathrm{TM}}$ Protein G Beads according to manufacturer's instructions (Promega, USA). Isotyping of mAbDi36/1 was performed using a commercial Kit (Sigma Isotyping kit ISO2, Sigma Aldrich, Switzerland) according to manufacturer's instructions and resulted in IgG1 subclass. 


\section{ELISAs}

Screening of mAbs (primary screening)

ELISAs have been performed in principle as described by Schucan et al. (2012). Briefly, plates were coated with $\sigma^{\prime \prime} / q$ D. immitis E/S (1:200 dilution in coating buffer: $0.1 \mathrm{M}$ carbonate/bicarbonate, $\mathrm{pH}$ 9.6) and $D$. immitis or $D$. repens adult somatic antigen (10 $\mu \mathrm{g} / \mathrm{ml}$ coating buffer) at $4{ }^{\circ} \mathrm{C}$ overnight in a humid chamber. If not stated otherwise, all following incubations were performed for one hour at $37^{\circ} \mathrm{C}$, followed by a four times washing step with ELISA wash buffer (physiological $\mathrm{NaCl}+0.3 \%$ Tween -20). After blocking ( $300 \mu \mathrm{l} /$ well) for $30 \mathrm{~min}$ utes with PBS-HT (containing PBS (pH 7.2) with $0.02 \%$ NaN3, $0.05 \%$ bovine haemoglobin (Fluka/ Sigma Aldrich, Switzerland) and 0.3\% Tween$20), \mathrm{mAb}$ containing supernatants in a 1:2 dilution in PBS-HT were added. Alkaline phosphatase labelled goat anti-mouse IgG (Sigma-Aldrich, Switzerland) in a 1:10'000 dilution in PBS-HT was used as conjugate. For enzymatic reaction, $100 \mu \mathrm{l} /$ well of a $1 \mathrm{mg} / \mathrm{ml}$ solution of 4-nitrophenylphosphate (Sigma-Aldrich, Switzerland) in $0.05 \mathrm{M}$ carbonate/bicarbonate buffer ( $\mathrm{pH}$ 9.8) containing $1 \mathrm{mM}$ $\mathrm{MgCl}$ were added and incubated for $10 \mathrm{~min}$ at 37 ${ }^{\circ} \mathrm{C}$. Absorbance values were read at $405 \mathrm{~nm}$ in a Multiscan RC ELISA reader (Thermo Labsystems, Finland).

\section{Specific antibody detection in dogs by}

\section{sandwich-ELISA}

ELISAs were performed using optimal concentrations of antigen, antibody and conjugate according to previous titration results. Test runs included a background and conjugate control. ELISA plates were coated with $5 \mu \mathrm{g}$ mAbDi36/1 per ml coating buffer and stored at $4{ }^{\circ} \mathrm{C}$ in a humid chamber overnight. After washing the plates four times with ELISA wash buffer, the plates were blocked (300 $\mu \mathrm{l} /$ well) with PBS-HT for 30 minutes at $37^{\circ} \mathrm{C}$. All following incubations were performed for one hour at $37^{\circ} \mathrm{C}$, followed by a four times washing step with ELISA wash buffer. D. immitis female somatic antigen $(5 \mu \mathrm{g} / \mathrm{ml}$ in PBS-HT; $100 \mu \mathrm{l} /$ well) was added. Sera were used in a standard dilution of 1:200 in PBS-HT $+10 \%$ fetal calf serum (100 $\mu \mathrm{l} /$ well). Subsequently, ReserveAP ${ }^{\mathrm{TM}}$ Phosphatase Labeled Goat anti-Dog IgG( $\gamma$ ) (Kirkegaard and Perry Lab. Inc., Gaithersburg, USA) in a 1:1250 dilution in PBS-HT was added $(100 \mu \mathrm{l} /$ well). Visualization of the reaction and OD-measurement was performed as stated above. Positive control samples from animals with proven infections and negative control samples from healthy dogs were included in all tests to adjust for plateto-plate variations.

\section{Statistical and data analysis}

Calculation of sensitivities was performed by dividing the number of seropositive animals by the total amount of infected animals, while specificity was calculated dividing the number of seronegative animals by the total number of uninfected animals tested. Exact binominal 95\% confidence intervals (CI) for means of binominal variables were calculated with unweighted data according to Clopper and Pearson (1934). The cut-off value was calculated as mean plus 3 standard deviations of the ELISA OD A $405 \mathrm{~nm}$ values of sera from 34 healthy dogs from a known filarial non-endemic region. Graphics were generated using Excel 2010 (Microsoft Corporation, Redmond, US).

\section{Results}

The mAb against an epitope of crude adult D. immitis antigen, designated 'mAbDi36/1' (IgG1 isotype), allowed the detection of filarial infection in dog serum samples.

\section{Sensitivity and specificity}

Sera of dogs naturally infected with Dirofilaria spp. or other parasites as well as sera from healthy dogs were used to determine the diagnostic values of the ELISA. Values on sensitivity and specificity are provided in Table 1. Sensitivity of the assay for 
Table 2 Cross-reactivity of the ELISA in sera of dogs infected with nematodes other than Dirofilaria spp. (Cut-off determination see Table 1).

\begin{tabular}{|l|c|c|c|}
\hline Parasite species & number of dogs (n) & Positive dogs (n) & $\begin{array}{c}\text { Positive dogs (\%) } \\
(95 \% \text { Cl) }\end{array}$ \\
\hline Acanthocheilonema reconditum* & 10 & 5 & $50.0 \%(18.7-81.3)$ \\
\hline Dipetalonema drunculoides* & 9 & 6 & $66.7 \%(29.9-92.5)$ \\
\hline Capillaria aerophilia* $^{*}$ & 7 & 1 & $14.3 \%(0.4-57.9)$ \\
\hline Trichuris vulpis\# & 2 & 0 & $0 \%(0.0-77.6)$ \\
\hline Ancylostoma caninum\# & 4 & 0 & $0 \%(0.0-52.7)$ \\
\hline Crenosoma vulpis* $^{*}$ & 6 & 1 & $16.7 \%(0.4-64.1)$ \\
\hline Toxocara canis\# & 5 & 0 & $0 \%(0.0-45.1)$ \\
\hline Angiostrongylus vasorum & & 0 & $0 \%(0.0-25.9)$ \\
\hline Angiostrongylus vasorum* & 10 & 1 & $14.3 \%(0.4-57.9)$ \\
\hline
\end{tabular}

*naturally infected dogs; \#experimentally infected dogs; $\mathrm{Cl}$ : Confidence interval

D. immitis patent infections was calculated to be 93.8\% (95\% CI: 79.2-99.2\%), whereas sensitivity on patent $D$. repens-infections reached $100 \%(95 \%$ CI: 81.9-100\%). Specificity was determined to be $98.6 \%$ (95\% CI: $92.2-100 \%)$ with sera of 69 dogs from a non-endemic area.

Sera of experimentally and naturally helminthinfected dogs were analyzed by the presented ELISA to determine potential cross-reactions. Results are provided in Table 2. No positive ELISA results were obtained with sera of dogs with patent infections of T. vulpis, A. caninum and T. canis. Cross-reactions were evidenced in $50 \%$ and $66.7 \%$ of dogs naturally infected with $A$. reconditum and $D$. dracunculoides, respectively. Sera of dogs naturally infected with $C$. aerophila (syn. Eucoleus aerophilius) cross-reacted in $14.3 \%$ of cases, sera of C. vulpis naturally infected dogs in $16.7 \%$ and sera of $A$. vasorum naturally infected dogs in $14.3 \%$, whereas sera of $A$. vasorum experimentally infected dogs resulted negative in ELISA.

\section{Detection of antibodies during experimen-} tal $D$, repens infections

Results are illustrated in Figure 1. Seroconversion of experimentally infected dogs was demonstrated between 24 and 80 dpi. The earliest antibody detection took place between 24 and 52 dpi in one infected dog (ID 108). Antibody levels increased until 164-178 dpi, before they reached an almost constant plateau around 220 dpi until necropsy at 406 dpi.

\section{Discussion}

The development of several ELISAs for serological antibody diagnosis of heartworm disease in dogs was initiated in the early $1980^{\text {th }}$ (Grieve et al. 1981, Sisson et al. 1985, Grieve and Knight 1985). ELISAs were designed to detect antibodies against purified somatic antigen of adult $D$. immitis and infected dogs became antibody positive as early as 4 months after single or repeated inoculations with L3. Furthermore, the antibody response persisted for at least 6 months following adulticidal treatment or natural death of the adult heartworms (Grieve et al. 1981). In the present study, antibodies in experimentally $D$. repens infected dogs could be detected earlier between 24 and 80 days post inoculation (dpi) and remained on a high level during the course of the study until necropsy at 
$406 \mathrm{dpi}$. These results suggest that the present test detects antibodies directed against larval and adult stages of $D$. repens in dogs. It is highly likely that similar courses of antibody development also occur in D. immitis-infected dogs. However, further studies with sera of experimentally $D$. immitis-infected dogs during course of infection need to confirm this assumption.

Considerable problems with previous antibody tests include their poor specificity due to homologous helminth interspecies epitopes. Previous assays based on the use of adult somatic $D$. immi$t i s$ antigens have therefore provided false-positive results (cross-reactions), when dogs are infected with Toxocara canis (Grieve et al. 1981, Ott et al. 1985, Thilsted et al. 1987), Angiostrongylus vasorum or Acanthocheilonema spp. and Dipetalonema spp. (Maier and Deplazes, personal communications). In the presented ELISA, cross-reactions were observed in $50 \%$ and $66.7 \%$ of sera from dogs naturally infected with $A$. reconditum and D. dracunculoides, respectively. Sera of these dogs were originated from Dirofilaria endemic regions such as Italy, Spain and Portugal and exposure of these dogs with Dirofilaria spp. cannot be ruled out. However, due to the close taxonomic relation of the genus Acanthocheilonema/Dipetalonema and Dirofilaria, cross-reactions between these genera are most probable. Nonetheless, Acanthocheilonema/Dipetalonema spp. are not endemic in hitherto Dirofilaria spp. non-endemic regions, where the present test is recommended to screen large dog populations. Cross-reactions were not observed with sera of dogs experimentally infected with T. canis, T. vulpis, A. vasorum and A. caninum, whereas one dog of each group naturally infected with $A$. vasorum (14.3\%), C. vulpis (16.7\%) and C. aerophila (14.3\%) showed a positive serological reaction. Nevertheless, all the last mentioned positive dogs from this study were naturally infected with helminths and particularly in case of $C$. aerophila when dogs were originated from Italy, potential multi-infections with various filariae leading to positive results in the presented
ELISA cannot completely be excluded. To increase the specificity of the presented ELISA, somatic D. immitis antigen was on-plate purified with a filarial-specific $\mathrm{mAb}$ resulting in a highly specific sandwich-ELISA. The use of native antigens of adult $D$. immitis represents an easy way of antigen preparation and the (on-plate) antigen purification step insures a high reproducibility of the ELISA as experienced with a similar test by Schucan et al. (2012).

Poor Sensitivity around $85 \%$ was observed in earlier serological ELISA approaches for the detection of antibodies against $D$. immitis somatic antigen (Glickmann et al. 1984, Thilsted et al. 1987). In contrast, sensitivities of the presented ELISA were very high in Dirofilaria-infected dogs (D. immitis $93.8 \%$; D. repens $100 \%)$. However, for the confirmation of these data, more field studies have to be performed with naturally infected hosts including occult infected animals and critically analyzed in different endemic areas. Furthermore, a positive ELISA indicates possible parasite-exposure, but not necessarily active heartworm or skin worm infection.

The here presented ELISA demonstrated its potential for use on a large scale initial screening in epidemiological or surveillance studies, best in combination with other serological tests for nematode infections for the documentation of filarial transmission in low- and non-endemic regions.

\section{Funding}

This research was financed by funds of the Institute of Parasitology, University of Zurich.

\section{Conflict of interest}

The authors declare that they have no competing interests.

\section{Ethicall standards}

All applicable international and institutional guidelines for the care and use of animals were followed. Mice experiments were conducted according to the Swiss guidelines for animal experimentation 
and approved by the Cantonal Veterinary Office of Zurich prior to the study (permission number 139/2015).

\section{Open access}

This article is distributed under the terms of the Creative Commons Attribution 4.0 International
License (http://creativecommons.org/licenses/ by/4.0/), which permits unrestricted use, distribution, and reproduction in any medium, provided you give appropriate credit to the original author(s) and the source, provide a link to the Creative Commons license, and indicate if changes were made.

\section{References}

Atkins CE (2003) Comparison of results of three commercial heartworm antigen test kits in dogs with low heartworm burdens. J Am Vet Med Assoc 222: 1221-1223

Baneth G, Thamsborg SM, Otranto D, Guillot J, Blaga R, Deplazes P, Solano-Gallego L (2016) Major parasitic zoonoses associated with dogs and cats in Europe. J Comp Pathol 155: $54-74$

Barutzki D, Schaper R (2009) Natural infections of Angiostrongylus vasorum and Crenosoma vulpis in dogs in Germany (2007-2009). Parasitol Res 105: 39-48

Bell D (1967) Membrane filters and microfilariae: a new diagnostic technique. Ann trop Med Parasitol 61:220-223

Bowman DD, Atkins CE (2009) Heartworm biology, treatment, and control. Vet Clin North Am Small Anim Pract 39: $1127-1158$

Bowman DD, Mannella C (2011) Macrocyclic lactones and Dirofilaria immitis microfilariae. Top Companion Anim Med 26: $160-172$

Cancrini G, Scaramozzino P, Gabrielli S, Di Paolo M, Toma L, Romi R (2007) Aedes albopictus and Culex pipiens implicated as natural vectors of Dirofilaria repens in central Italy. J Med Entomol 44: 1064-1066

Chalifoux L, Hunt RD (1971) Histochemical differentiation of Dirofilaria immitis and Dipetalonema reconditum. J Am Vet Med Assoc 158: 601-605

Clopper CJ, Pearson ES (1934) The use of confidence or fiducial limits illustrated in the case of the binomial. Biometrika 26: $404-413$

Ciucă L, Genchi M, Kramer L, Mangia C, Miron LD, Prete LD, Maurelli MP, Cringoli G, Rinaldi L (2016) Heat treatment of serum samples from stray dogs naturally exposed to Dirofilaria immitis and Dirofilaria repens in Romania. Vet Parasitol 225: $81-85$
Deplazes P, Smith NC, Arnold P, Lutz H, Eckert J (1995) Specific IgG1 and IgG2 antibody responses of dogs to Leishmania infantum and other parasites. Parasite Immunol 17: $451-458$

Di Cesare A, Otranto D, Di Giulio E, Simonato G, Latrofa MS, La Torre F, Coccia G, Traversa D (2013) Microfilarial periodicity of Dirofilaria repens in naturally infested dogs. Parasitol Res 112: 4273-4279

Drake J, Gruntmeir J, Merritt H, Allen L, Little SE (2015) False negative antigen tests in dogs infected with heartworm and placed on macrocyclic lactone preventives. Parasit Vectors 8: 68

Genchi C, Kramer LH, Rivasi F (2011) Dirofilarial infections in Europe. Vector Borne Zoonotic Dis 11:1307-1317. doi: 10.1089/vbz.2010.0247

Glickman LT, Grieve RB, Breitschwerdt EB, Mika-Grieve M, Patronek GJ, Domanski LM, Root CR, Malone JB (1984) Serologic pattern of canine heartworm (Dirofilaria immitis) infection. Am J Vet Res 45: 1178-1183

Goodwin JK (1998) The serologic diagnosis of heartworm infection in dogs and cats. Clin Tech Small Anim Pract 13: $83-87$

Grieve RB, Mika-Johnson M, Jacobson RH, Cypess RH (1981) Enzyme-linked immunosorbent assay for measurement of antibody responses to Dirofilaria immitis in experimentally infected dogs. Am J Vet Res 42: 66-69

Grieve RB, Knight DH (1985) Anti-Dirofilaria immitis antibody levels before and after anthelmintic treatment of experimentally infected dogs. J Parasitol 71: 56-61

Knott J (1939) A method for making microfilarial survey on day blood. Trans R Soc Trop Med Hyg 33: 191-196

Latrofa MS, Dantas-Torres F, Annoscia G, Genchi M, Traversa D, Otranto D (2012) A duplex real-time polymerase chain reaction assay for the detection of and differentiation between Dirofilaria immitis and Dirofilaria repens in dogs and mosquitoes. Vet Parasitol 185: 181-185 
Lee AC, Bowman DD, Lucio-Forster A, Beall MJ, Liotta JL, Dillon R (2011) Evaluation of a new in-clinic method for the detection of canine heartworm antigen. Vet Parasitol 177: $387-391$

Little SE, Munzing C, Heise SR, Allen KE, Starkey LA, Johnson EM, Meinkoth J, Reichard MV (2014) Pre-treatment with heat facilitates detection of antigen of Dirofilaria immitis in canine samples. Vet Parasitol 203: 250-252

Ludlam KW, Jachowski LA Jr, Otto GF (1970) Potential vectors of Dirofilaria immitis. J Am Vet Med Assoc 157: 1354-1359

Magnis J, Lorentz S, Guardone L, Grimm F, Magi M, Naucke TJ, Deplazes P (2013) Morphometric analyses of canine blood microfilariae isolated by the Knott's test enables Dirofilaria immitis and D. repens species-specific and Acanthocheilonema (syn. Dipetalonema) genus-specific diagnosis. Parasit Vectors 6: 48

Ott RA, Staples M, Weekley M, Maggio ET (1985) Demonstration of both immunologically unique and common antigenic determinants in Dirofilaria immitis and Toxocara canis using monoclonal antibodies. Vet Immunol Immunopathol 10: 147-153

Peribáñez MA, Lucientes J, Arce S, Morales M, Castillo JA, Gracia MJ (2001) Histochemical differentiation of Dirofilaria immitis, Dirofilaria repens and Acanthocheilonema dracunculoides microfilariae by staining with a commercial kit, Leucognost-SP. Vet Parasitol 102: 173-175

Petry G, Genchi M, Schmidt H, Schaper R, Lawrenz B, Genchi C (2015) Evaluation of the adulticidal efficacy of imidacloprid $10 \% /$ moxidectin $2.5 \%(\mathrm{w} / \mathrm{v})$ spot-on (Advocate ${ }^{\circledR}$, Advantage ${ }^{\circledR}$ Multi) against Dirofilaria repens in experimentally infected dogs. Parasitol Res 114: 131-144

Rawlings CA, Dawe DL, McCall JW, Keith JC, Prestwood AK (1982) Four types of occult Dirofilaria immitis infection in dogs. J Am Vet Med Assoc 180: 1323-1326

Rishniw M, Barr SC, Simpson KW, Frongillo MF, Franz M, Dominguez Alpizar JL (2006) Discrimination between six species of canine microfilariae by a single polymerase chain reaction. Vet Parasitol 135: 303-214
Schnyder M, Tanner I, Webster P, Barutzki D, Deplazes P (2011) An ELISA for sensitive and specific detection of circulating antigen of Angiostrongylus vasorum in serum samples of naturally and experimentally infected dogs. Vet Parasitol 179: $152-158$

Schnyder M, Deplazes P (2012) Cross-reactions of sera from dogs infected with Angiostrongylus vasorum in commercially available Dirofilaria immitis test kits. Parasit Vectors 5: 258

Schucan A, Schnyder M, Tanner I, Barutzki D, Traversa D, Deplazes P (2012) Detection of specific antibodies in dogs infected with Angiostrongylus vasorum. Vet Parasitol 185: $216-224$

Silaghi C, Beck R, Capelli G, Montarsi F, Mathis A (2017) Development of Dirofilaria immitis and Dirofilaria repens in Aedes japonicus and Aedes geniculatus. Parasit Vectors 10: 94

Sisson D, Dilling G, Wong MM, Thomas WP (1985) Sensitivity and specificity of the indirect-fluorescent antibody test and two enzyme-linked immunosorbent assays in canine dirofilariasis. Am J Vet Res 46: 1529-1533

Thilsted JP, Whorton J, Hibbs CM, Jillson GP, Steece R, Stromei M (1987) Comparison of four serotests for the detection of Dirofilaria immitis infection in dogs. Am J Vet Res 48: $837-841$

Traversa, D, Latrofa MS, Di Cesare A, Meloni S, Castagna S, Otranto D (2011) Phylogenesis of Capillaria aerophilia (Trichocephalida, Trichuridae) from different hosts and countries. In: Proceedings of the $23^{\text {rd }}$ Conference of the WAAVP, Buenos Aires, Argentina, 21-24 August 2011

Velasquez L, Blagburn BL, Duncan-Decoq R, Johnson EM, Allen KE, Meinkoth J, Gruntmeir J, Little SE (2014) Increased prevalence of Dirofilaria immitis antigen in canine samples after heat treatment. Vet Parasitol 206: 67-70

Weil GJ (1987) Dirofilaria immitis: identification and partial characterization of parasite antigens in the serum of infected dogs. Exp Parasitol 64: 244-251 
